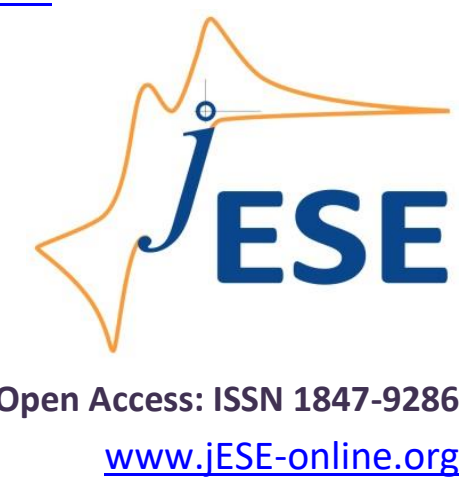

Review

\title{
Chemisorption as the essential step in electrochemical energy conversion
}

\author{
Ana S. Dobrota, Igor A. Pašti ${ }^{凶}$
}

University of Belgrade - Faculty of Physical Chemistry, Studentski trg 12-16, 11158 Belgrade, Serbia Corresponding author: ${ }^{{ }_{\text {igor }} @ f f h . b g . a c . r s ;}$; Tel.: +381-11-3336-625; Fax: +381-11-2187-133

Received: October 31, 2019; Accepted: January 6, 2020

\begin{abstract}
Growing world population and energy demands have placed energy conversion and storage into the very centre of modern research. Electrochemical energy conversion systems including batteries, fuel cells, and supercapacitors, are widely considered as the next generation power sources. Even though they rely on different mechanisms of energy conversion and storage, fundamentally these are all electrochemical cells, operating through processes taking place at the solid/liquid interfaces, i.e. electrodes. Considering the interfacial nature of electrodes, it is clear that adsorption phenomena cannot be neglected when considering electrochemical systems. More than that, they are of crucial importance for electrochemical processes and represent an essential step in electrochemical energy conversion. In this contribution we give an overview of the phenomena underlying the operation of sustainable metal-ion batteries, fuel cells and supercapacitors, ranging from electrocatalytic reactions and pseudo-faradaic processes to purely adsorptive processes, emphasizing the types, roles and significance of chemisorption. We review experimental and theoretical methods which can provide information about chemisorption in the mentioned systems, stressing the importance of combining both approaches.
\end{abstract}

\section{Keywords}

Adsorption; reactivity trends; electrocatalysis; electrochemical power sources.

\section{Introduction}

The surface of the material is its outlook to the external environment. Therefore, many problems associated with modern materials can be solved by understanding the physical and chemical interactions that occur at materials interfaces. The nature and the state of the surface influence many properties of the material, including corrosion rate, catalytic activity, adhesive properties, wettability, contact potential, and others. These characteristics can be tuned by surface modification, for example surface functionalization or doping. Let us look at a freshly prepared solid 
surface. If this surface is surrounded by a gaseous or liquid phase, it is exposed to the molecules of that phase. As a molecule approaches to a surface, its electron density redistributes in such a way that maximizes bonding to that surface. Under normal conditions, this will result in rather quick coverage of the surface with these molecules, i.e. adsorption will occur. Adsorption is an increase of the concentration of a substance at the interface of two phases. In electrochemistry, this is usually related to the solid|liquid interface. Adsorption energy is a key quantity describing the strength of the interaction of molecules with the surface. While on single crystal surfaces, several high symmetry sites are available for adsorption, the preferential adsorption site is the one with the most exothermic adsorption. The way in which electron density redistributes in this process is determined by electronic structures of the surface and the adsorbate. Depending on the type of forces responsible for adsorption, we can divide it into chemical and physical adsorption. Chemical adsorption (chemisorption) is, according to IUPAC, adsorption which results from chemical bond formation (strong interaction) between the adsorbent and the adsorbate in a monolayer on the surface [1]. The forces responsible for chemisorption are the same kind as those that lead to the formation of chemical compounds. On the other hand, physical adsorption (physisorption, van der Waals adsorption) is adsorption in which the forces involved are weak van der Waals forces, which include dipole-dipole, dipole-induced dipole and London (instantaneous induced dipole-induced dipole) forces. They do not involve a significant change in the electronic orbital patterns of the species involved $[2,3]$. The problem of distinguishing between chemisorption and physisorption is the same as between chemical and physical interactions in general. The dividing line between the two is not completely sharp, and intermediate cases exist, e.g. adsorption involving strong hydrogen bonds, or weak charge transfer. However, chemisorption can be identified using some of the following criteria: (i) it is chemically specific (variations between substrates of different chemical composition, and between different surface planes of the same crystal); (ii) it leads to changes in the electronic structure of the adsorbate and adsorbent; (iii) the chemisorption elementary step often requires an activation energy; (iv) it is often dissociative (adsorbate dissociates into two or more fragments, both or all of which are bound to the surface of the adsorbent), and may not be reversible; (v) just one monolayer of adsorbate is usually formed. In general, chemisorption properties are determined by the compatibility of functionalities of adsorbents and adsorbates.

Now, let us look at an electrochemical system. Each such system consists out of at least two pieces of a solid electronic conductor in electrical contact with each other through the electrolyte, from one side, and through an outer electronic conductor, from the other side of the cell. In such a way a closed electrical circuit is formed. The interface between the solid electronic conductor and the electrolyte (usually liquid ionic conductor) is the electrode of an electrochemical cell. Considering the interfacial nature of the electrode, it is clear that adsorption phenomena are always taking place at the electrode and are of crucial importance for electrochemical processes and represent their essential step.

The ever-growing energy demands have placed energy conversion and storage into the very centre of modern research. Electrochemical energy conversion systems including batteries, fuel cells, and supercapacitors, are widely considered as the next generation power sources. Even though they rely on different mechanisms of energy conversion and storage, fundamentally these all are electrochemical systems, operating through processes taking place at the electrodes.

In batteries and fuel cells electrical energy is generated through Faradaic processes, i.e. through redox reactions (electron transfer) at the electrodes. While the inside of a battery contains all that is needed for its operation, fuel cells obtain the electroactive species (species which undergo redox 
reactions) from outside of the cell (in the case of hydrogen fuel cells - oxygen from air and hydrogen from external storage unit), whereas the solid part of the electrode is just charge-transfer media (not changed in the overall reaction). On the other hand, electrochemical capacitors (supercapacitors, ultracapacitors) can be classified into two categories based on their energy storage mechanism: (i) electrical double layer (EDL) capacitors, in which the capacitance is the result of pure electrostatic accumulation and disorganization of charges at the electrode, strongly dependent on the surface area of the electrode materials accessible to the electrolyte ions; (ii) pseudo-capacitors, in which fast and reversible faradic processes take place [4]. These two mechanisms can work simultaneously depending on the nature of electrode materials.

Electrochemical reactions take place at the electrode, i.e. at the solid/liquid interface. Therefore, it is clear that charge transfer processes in some types of batteries and, generally, fuels cells represent cases of heterogeneous catalysis, in which the process rate is affected by the presence of a solid surface which mediates chemical transformations. During every catalytic cycle many bonds are cleaved and formed, but in the end the catalyst remains unchanged. A special case of heterogeneous catalysis in which the rate of the process is additionally influenced by the electrode potential and the electrode material properties is electrocatalysis. In this sense, virtually every electrochemical reaction is fundamentally electrocatalytic [5], excluding outer sphere reactions. For a Faradaic electrochemical process to take place, at least three following steps have to occur: (i) transfer of electroactive species (reactant) to the electrode, (ii) charge transfer at the electrode, resulting in chemical transformation of the reactant to the product and (iii) transfer of the product away from the electrode. However, in majority of electrochemical reactions, interactions of reactants with the electrode or formation of adsorbed intermediates is the prerequisite for charge transfer. For example, this is the case for one of the most important electrochemical reactions, hydrogen evolution reaction (HER), where adsorbed atomic hydrogen $\left(\mathrm{H}_{\mathrm{ads}}\right)$ is formed in the process, in overall described as: $2 \mathrm{H}^{+}+2 \mathrm{e}^{-} \rightarrow \mathrm{H}_{2}$.

An essential step in every heterogeneous catalytic reaction, and therefore in electrochemical energy conversion processes, is the chemical bond formation between the catalyst surface and the reactant/intermediate, i.e. chemisorption. In general, chemisorption properties are determined by the combination of electronic structures of the substrate and the adsorbate. As an adsorbate molecule approaches a surface, redistribution of electron density might result in weakening of some of the bonds inside the adsorbate molecule. In that way the solid catalyst activates the adsorbate, making it prone to chemical changes. The interaction of the reactants/intermediates with the catalyst has to be optimal in strength (Sabatier principle, Figure 1): if too weak, the reactants will not bind to the catalyst and the reaction will not be able to take place [6]. On the other hand, if the interaction is too strong, the catalyst active sites will be blocked by reactant, intermediate or product molecules, leaving no active sites available for new reactant molecules which would continue the reaction.

In this contribution, we address chemisorption as the initial and fundamental step in electrochemical energy conversion and storage systems. We explore which adsorbates and adsorbents are of electrochemical interest and review experimental and theoretical methods used for exploring such systems, demonstrating how electronic structure calculations can provide fundamental, atomic-level understanding of the interaction of different types of adsorbates with various substrates of great importance in electrochemical energy conversion. We emphasize the importance of combining experimental and theoretical approaches in order to complete the adsorption puzzle. 


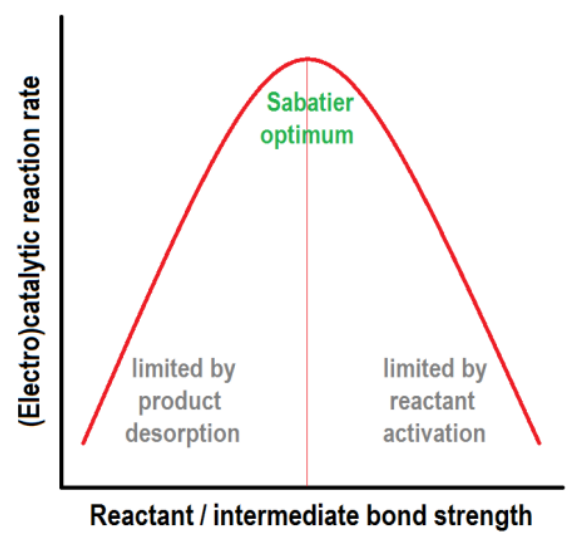

Figure 1. Schematic view of the Sabatier principle: finding the optimal bond strength.

\section{Adsorbates of electrochemical interest}

Carbon monoxide oxidation ( $\mathrm{CO}+1 / 2 \mathrm{O}_{2} \rightarrow \mathrm{CO}_{2}$ ) is one of the simplest catalytic reactions, used as a prototype for understanding the oxidation of small organic molecules (e.g. methanol, ethanol, formic acid) used as the fuel in some fuel cells. It is also of great interest for the control of exhaust gases, air purification and sensors. Three major types of $\mathrm{CO}$ oxidation catalysts are used: platinum group metallic catalysts (whose surface might be oxidized), bulk oxide catalysts (e.g. $\mathrm{CeO}_{2}$ ), and oxide-supported metal clusters. Therefore, their interaction with $\mathrm{CO}$ molecule is of great practical interest.

Hydrogen fuel cells are a perspective replacement for fossil fuels which would diminish atmospheric pollution, giving water as the only by-product. In hydrogen fuel cells the oxygen reduction reaction (ORR) plays an essential role in determining the cell performance. Both in acidic and alkaline water environments ORR can proceed through two paths: (i) the direct ( $\left.4 \mathrm{e}^{-}\right)$pathway, and (ii) the indirect $\left(2 \mathrm{e}^{-}\right)$pathway, as given in Table 1. Due to higher Faradaic efficiency, the direct $4 \mathrm{e}^{-}$pathway is preferable. However, the mechanism of this pathway consists of a number of steps in which molecular oxygen has to dissociate at the surface and recombine with hydrogen ions to form water. Therefore, dissociative chemisorption of $\mathrm{O}_{2}$ can be considered as a crucial step in ORR. Looking at the anode side, different fuels can be oxidized, starting with $\mathrm{H}_{2}$ or small organic molecules. In all the cases, anode reactions involve formation of adsorbed intermediates.

Another electrochemical reaction of great interest for hydrogen fuel cells is hydrogen evolution reaction (HER). Hydrogen is considered to be the future energy carrier. Inside a fuel cell hydrogen undergoes oxidation, giving water as the final product, but there is the issue of providing and storing the hydrogen fuel in the first place. In acidic environment (Table 1) the first HER step is proton reduction, yielding adsorbed hydrogen (Volmer reaction: $\mathrm{H}^{+}+\mathrm{e}^{-} \rightarrow \mathrm{H}_{\text {ads }}$ ). Two of so adsorbed atoms can recombine on the surface, forming $\mathrm{H}_{2}$ (Tafel reaction: $2 \mathrm{H}_{\text {ads }} \rightarrow \mathrm{H}_{2}$ ), or one $\mathrm{H}_{\text {ads }}$ can undergo electrochemical desorption (Heyrovsky reaction: $\mathrm{H}_{\mathrm{ads}}+\mathrm{H}^{+}+\mathrm{e}^{-} \rightarrow \mathrm{H}_{2}$ ). Since $\mathrm{H}_{\text {ads }}$ formation is the first step in this mechanism, adsorption energy of atomic hydrogen is a valuable parameter for predicting material's activity for HER and $\mathrm{H}_{2}$ storage capability.

Table 1. Oxygen reduction and hydrogen evolution pathways in acidic and alkaline solutions.

\begin{tabular}{|c|c|c|}
\hline Reaction/pathway & Acidic environment & Alkaline environment \\
\hline direct ORR (4e-) & $\mathrm{O}_{2}+4 \mathrm{H}^{+}+4 \mathrm{e}^{-} \rightarrow 2 \mathrm{H}_{2} \mathrm{O}$ & $\mathrm{O}_{2}+2 \mathrm{H}_{2} \mathrm{O}+4 \mathrm{e}^{-} \rightarrow 4 \mathrm{OH}^{-}$ \\
\hline indirect ORR ( $\left.2 \mathrm{e}^{-}\right)$ & $\begin{array}{c}\mathrm{O}_{2}+2 \mathrm{H}^{+}+2 \mathrm{e}^{-} \rightarrow \mathrm{H}_{2} \mathrm{O}_{2} \\
\mathrm{H}_{2} \mathrm{O}_{2}+2 \mathrm{H}^{+}+2 \mathrm{e}^{-} \rightarrow 2 \mathrm{H}_{2} \mathrm{O}\end{array}$ & $\begin{array}{c}\mathrm{O}_{2}+\mathrm{H}_{2} \mathrm{O}+2 \mathrm{e}^{-} \rightarrow \mathrm{HO}_{2}^{-}+\mathrm{OH}^{-} \\
\mathrm{HO}_{2}^{-}+\mathrm{H}_{2} \mathrm{O}+2 \mathrm{e}^{-} \rightarrow 3 \mathrm{OH}^{-}\end{array}$ \\
\hline HER & $2 \mathrm{H}^{+}+2 \mathrm{e}^{-} \rightarrow \mathrm{H}_{2}$ & $2 \mathrm{H}_{2} \mathrm{O}+2 \mathrm{e}^{-} \rightarrow \mathrm{H}_{2}+2 \mathrm{OH}^{-}$ \\
\hline
\end{tabular}


In the case of metal-ion batteries, the interaction of the chosen metal with the electrode material is of key interest, as they operate through insertion/removal of the given metal ions at the cathode/anode. Therefore, a key prerequisite for attaining high energy density (energy per unit volume) of a metal-ion battery is a cathode which accommodates and releases the metal ions quickly and in great capacity. Battery operation (charge/discharge cycles) assumes a chemical change with known Gibbs free energy change $(\Delta G)$, resulting in formation of well-defined phases. The open circuit potential of the cell can be related to $\Delta G$ of the reacting system. Most widely used today are lithiumion batteries. However, their sustainability is in question due to the limited lithium availability and consequent expected price increase. Therefore, intensive research efforts are made in the field of rechargeable metal-ion batteries involving more abundant light weight metals, such as sodium, magnesium, calcium, aluminium and zinc [7-10]. Due to their multivalent nature, which means that multiple electrons are transported during the electrochemical charge/discharge reactions, $\mathrm{Mg}, \mathrm{Ca}, \mathrm{Al}$ and $\mathrm{Zn}$ metal anodes can be used to obtain higher energy density, improved safety and lower initial and cycle-life costs than state-of-art lithium batteries [10]. That is why understanding the nature of the interaction of the mentioned metals with potential electrode materials is crucial for further development of rechargeable metal-ion batteries. Another important group of batteries are metal-air batteries, like lithium-air batteries, where metal lithium oxidizes on the anode, and oxygen undergoes reduction on the cathode, in the overall reaction: $2 \mathrm{Li}+\mathrm{O}_{2} \rightarrow \mathrm{Li}_{2} \mathrm{O}_{2}$. In this case, cleaving of the O-O bond is not necessary, resulting in improved kinetics and re-chargeability, and not requirements for Pt catalyst. However, metal-air batteries still face many practical problems restricting their massive production and use [7].

Another important question regarding adsorption is whether universal relationships between the binding energies of different adsorbates exist. Revealing such relationships would provide a simple tool for the assessment of surface reactivity towards various adsorbates using measurements with a single one. For some types of adsorbates and surfaces such interdependence has been demonstrated, as will be discussed later on.

\section{Understanding adsorption: experimental and theoretical tools united}

Each catalytic process involves multiple steps of bond breakage and formation. Capturing each of these steps is very difficult even by the finest of experimental techniques. That is where the electronic structure calculations become involved, demonstrating their true power and complementarity with experimental methods [11]. The computational and experimental approaches in catalysis should not be considered separately, because only by combining them one can obtain full understanding of the given catalytic process.

According to the classification given in ref. [12], experimental methods for investigating adsorption phenomena can be divided into three groups: (i) methods based on the measurement of changes in the electrical, magnetic and work function properties of the adsorbent during adsorption, (ii) methods based on the interaction between the adsorbate and radiation, electrons or ions, and (iii) spectroscopic methods. The first group includes measurements of the electrical conductivity of the adsorbent, Hall effect, magnetic properties, and the work function [12].

For exploring surface bonds, ultraviolet photoelectron spectroscopy (UPS) is often used [13]. UPS operates on the same principles as X-ray photoelectron spectroscopy (XPS), the only difference being that ultraviolet (instead of $\mathrm{X}$-ray) ionising radiation is used to induce the photoelectric effect. The spectra are obtained by irradiation of a solid surface with X-ray/UV light and measuring number and kinetic energy of electrons emitted from the material. As the energy of the ultraviolet photons 
is lower, most core level photoemissions are not accessible, and only the valence band region is probed. Due to the short inelastic mean free path of free electrons within a solid (determined by the properties of the solid through which they are travelling, and electrons kinetic energy), UPS is inherently surface sensitive, with an approximate information depth of 2-3 nm. Therefore, a signifycant fraction of the spectral contribution can be associated with the adsorbate. While UPS does not allow an atom-based view of bonding effects [14], X-ray emission spectroscopy (XES) and X-ray absorption spectroscopy (XAS) provide just that, through measurements of occupied/unoccupied densities of states [13-15]. XAS is a powerful tool, both element and site specific, for measuring the unoccupied density of electronic states of a material using high intensity X-ray radiation of tuneable energy. The fraction of X-ray photons absorbed by the samples electrons moving to the conduction band is measured, as their subsequent relaxation results in the emission of a photons having energy equal to the energy difference between the core level and excited states. XES is a complementary technique that probes the occupied density of electronic states of a material. Another experimental technique for determination of surface structures, which can be used both for qualitative and quantitative analysis, is low energy electron diffraction (LEED). In LEED, a beam of electrons of a well-defined low energy (typically $20-200 \mathrm{eV}$ ) is directed normally onto the sample. The diffraction pattern is formed by the elastically-scattered electrons, while secondary electrons are removed by energy-filtering grids. The analysis of the diffraction spot positions yields information on the size, symmetry and rotational alignment of the adsorbate unit cell with respect to the substrate unit cell. Single crystal adsorption calorimetry (SCAC) is a powerful method for measuring adsorption energies [16]. Microscopic methods such as scanning tunnelling microscopy (STM) and atomic force microscopy (AFM) can also provide valuable insights into the surface structures. STM is based on quantum tunnelling which occurs when the conducting tip scans the sample. While STM requires a conducting sample, AFM is appropriate for the insulating ones as well.

In contrast to experimental techniques, electronic structure methods provide atomic level resolution. However, the cost of these calculations requires introduction of significant approximations, and the results obtained cannot be considered as "absolute truth". Theoretical and experimental results should be taken together in order to have a picture as complete as possible. Two theoretical electronic structure methods have made the largest impact in the field of catalysis: (i) high-level $a b$ initio molecular orbital theory and (ii) density functional theory (DFT). DFT is based on two theorems given by Hohenberg and Kohn for the case of arbitrarily many electrons in a box subjected to external potential $u(r)[17,18]$ : (i) the system Hamiltonian and its ground state are uniquely determined by the electron density $(n(r))$, and (ii) an universal functional $(F[n])$ exists, which determines the total energy of the system as: $E[n]=\int u(r) n(r) \mathrm{d} r+F[n]$. The total energy has minimal value for the ground state $n(r)$. This way, the scalar electron density was used [19] instead of the many-body wavefunction. Kohn and Sham have divided this functional into the kinetic energy of non-interacting electrons, the energy of electron-electron interactions, and the exchange-correlation energy $\left(E_{\mathrm{xc}}\right)$. In this way, the uncertainty is reduced to $E_{\mathrm{xc}}$. Kohn-Sham self-consistent equations are solved iteratively, starting from a guessed $n(r)$, resulting in the total ground state energy of the system. The adsorption energy $\left(E_{\text {ads }}\right)$ can then be easily calculated as the difference in total energies between the final state (adsorbate adsorbed on the substrate) and the initial state (isolated adsorbate and substrate). Although $E_{\mathrm{xc}}$ is expected to be a small part of $F[n]$, it still makes DFT somewhat approximate, and not fully first-principles. To account for the unknown $E_{\mathrm{xc}}$, various approximations are used, including the local density approximation (LDA), generalized gradient approximation (GGA), hybrid functionals, and others. A proper choice of the electron exchange correlation functional is of great importance for accurate description of the chemi- 
sorption energetics [20]. Even though that due to exchange-correlation treatment DFT is more approximate than molecular orbital theory, it is more appealing for catalysis studies, as it offers the possibility of investigating more complex systems, closer to the real ones. Today, DFT is widely used as a numerical simulation tool for investigating systems consisting of up to $10^{2}-10^{3}$ atoms (depending on the available computational resources), for example for large molecules, solid bulks and interfaces. Moreover, its accuracy is high enough to explain and predict reactivity trends [11]. However, GGA results in the neglect of dispersion interactions. This approach leads to an incorrect description of physisorption, which can be of interest when looking at $\mathrm{O}_{2}$ and alkali metals adsorption on some materials, species of great importance for fuel cell and metal-ion batteries applications. In fact, in physisorbed systems LDA can give 'better' results than GGA [21], but dispersion can be included in GGA calculations through several corrections. Theoretical modelling can be used for rational catalyst design and capturing general trends and principles underlying various catalytic processes at the electronic/atomic level [11]. However, the electronic structure calculations are the most demanding computational approaches used in (electro)catalysis research. A detailed analysis of reaction mechanisms for a particular catalytic reaction on many possible substrates can be extremely timeconsuming and impractical. Additionally, for explicit treatment of an electrochemical interface, the existence of the solvent and the electrode potential would have to be taken into account, but there is no proper scheme to theoretically account for the electrode potential at the level which is affordable enough to allow routine use of such approach [22]. Luckily, it seems that in most cases, the treatment of electrochemical reactions from the aspect of the electrocatalysis does not require an inclusion of the electrode potential. The presence of a solvent can also be disregarded, or included implicitly through corrective factors of the adsorption energies of the reaction reactants/intermediates [23]. This way, electrocatalytic reactions can be theoretically treated as reactions at the solid/gas interface. However, there is an enormous number of possibilities when it comes to systems which could be treated theoretically. Therefore, it is practical to use some empirical and semi-empirical filters, e.g. element abundance, to reduce the number of systems requiring explicit DFT treatment. An example of such pre-screening can be found in ref. [24].

Microscopic properties of (electro)catalytic materials which can be associated with their performance according to kinetics of (electro)catalytic processes are called catalytic activity descriptors. They can be obtained by experimental and theoretical techniques and used for tuning the catalytic activity of a given material and establishing design concepts in search for new catalysts [25]. Since in heterogeneous catalysis the interaction between the catalyst and reactants/ intermediates plays the paramount role for the catalytic performance, these descriptors are often corresponding adsorption energies, as will be shown later on.

\section{Chemisorption on transition metal surfaces}

Chemisorption trends on transition metal surfaces have been highly elaborated due to their significance in heterogeneous catalysis. For example, transition metal-based electrodes are typically used as catalysts for HER in alkaline medium, while Pt-group metals are the best catalysts for hydrogen and oxygen electrode reactions.

Hammer and Nørskov have formulated a concept, known as the $d$-band model, which describes adsorption trends on transition metal surfaces using the position of the metals $d$-band [26]. This model provides a link between the electronic structure and the reactivity of transition metal surfaces, as well as their alloys and overlayers. The key parameter determining the adsorption strength, $d$-band centre, can be obtained both experimentally (from spectroscopical methods) and 
by theoretical calculations [27-29]. According to $d$-band model, interaction of an adsorbate with the metal surface can be thought as a two-step process. First, the electronic state of the adsorbate widens due to its interaction with the metal wide, half-filled s-states (which are the same for all transition metals). Next, the so-widened adsorbate state interacts with metal narrow $d$-state, giving rise to bonding and anti-bonding states. Their relative occupancies, determined by the positions of the adsorbate state and the metal $d$-states, dictate the strength of the overall interaction. In order for all of this to occur, the adsorbate atom over which the bonding is to take place should have a radical state, i.e. an unpaired electron [30]. If not, rehybridization is needed to form such a radical state enabling the interaction with the surface [30]. Of course, there are other scenarios as well.

If instead of unpaired electrons the adsorbate possesses an unsaturated $\pi$-electron system (e.g. $\mathrm{N}_{2}, \mathrm{O}_{2}, \mathrm{CO}$, or unsaturated hydrocarbon molecules), binding to a metal surface is possible via virtual radical state created by partial mixing or excitation of the bonding and antibonding $\pi$ and $\pi^{*}$ orbitals [31]. The mechanism of the virtual radical state formation depends on the $\pi \rightarrow \pi^{*}$ excitation energy and determines the final adsorption geometry. Complete cleavage of one $\pi$ bond inside the adsorbate would leave both of the involved atoms "radicalized", prone to bonding to the metal surface, resulting in horizontal adsorption. However, such bond cleavage would mean fully populating $\pi^{*}$ orbital, which is not favourable if $\pi \rightarrow \pi^{*}$ excitation energy is relatively big (compared to the energy that would be obtained upon bonding). Therefore, $\pi$-unsaturated molecules with smaller excitation energies, such as unsaturated hydrocarbons, will adsorb in parallel to the surface. This kind of bonding is explained in DCD (Dewar, Chatt and Duncanson) model as donation of charge from the molecule highest occupied $\pi$-orbital into the metal, and a subsequent back-donation from filled metal states into the lowest unoccupied $\pi^{*}$-orbital $[32,33]$. On the other hand, molecules with higher excitation energies (e.g. $\mathrm{N}_{2}, \mathrm{CO}$ ) will not undergo full bond breakage, $\pi^{*}$ will not be fully occupied, one atom will be more activated, and the molecule will adsorb vertically via that atom to the surface. For molecules that cannot engage in bonding neither through broken bonds nor virtually via $\pi$-electron system, rehybridization requires too much energy (compared to the energy obtained upon bonding) resulting in weak overall interaction with the surface. That is the case with molecules with lone pair electrons in rigid $\sigma$ orbitals, e.g. $\mathrm{H}_{2} \mathrm{O}$. The situation is similar for the case of saturated hydrocarbons, in which only small polarization rearrangements of the molecular orbital structure are energetically possible, resulting in weak physisorption [30].

Regarding $\mathrm{CO}$ oxidation on densely packed transition metal surfaces, three adsorption mechanisms have been proposed: (i) Langmuir-Hinshelwood [34], (ii) Eley-Rideal [11] and (iii) Marsvan Krevelen mechanism [35,36]. Langmuir-Hinshelwood model is most widely accepted and assumes adsorption of both $\mathrm{CO}$ and $\mathrm{O}_{2}$ on the catalyst surface, but the adsorption of $\mathrm{O}_{2}$ is dissociative, yielding oxygen adatoms: $\mathrm{O}_{2} \rightarrow 2 \mathrm{O}_{\text {ads. }}$. Thus, adsorbed $\mathrm{CO}$ and $\mathrm{O}_{\text {ads }}$ form $\mathrm{CO}_{2}$ which desorbs from the surface (Figure 2a).

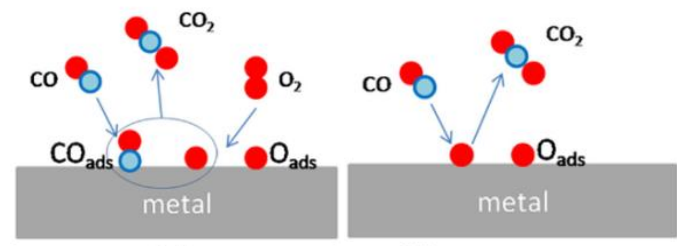

(a)

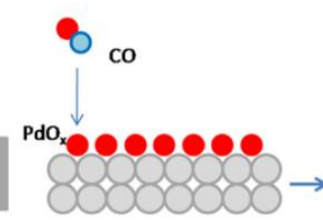

(b)

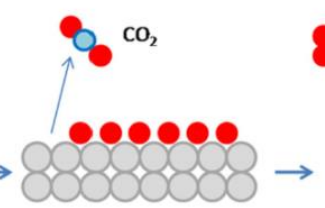

(c)

Figure 2. Different mechanisms of $\mathrm{CO}$ oxidation on platinum group metal surface:

(a) Langmuir-Hinshelwood mechanism of $\mathrm{CO}$ and $\mathrm{O}_{2}$ co-adsorption, (b) Eley-Rideal mechanism of just $\mathrm{O}_{2}$ adsorption, and (c) Mars-van Krevelen mechanism for oxidized metal surfaces.

Reprinted from ref. [11], ${ }^{\circ}$ 2014, Akadémiai Kiadó, Budapest, Hungary 
Within Eley-Rideal mechanism, CO does not adsorb, but rather reacts from the gas phase with $\mathrm{O}_{\text {ads }}$ on the catalyst surface (Figure $2 \mathrm{~b}$ ). If the metal surface is oxidized, the lattice $\mathrm{O}$ atoms of the first layer can be used for $\mathrm{CO}$ oxidation (Figure $2 \mathrm{c}$ ). This results in vacancy formation and subsequent surface healing by $\mathrm{O}_{2}$ from the gas phase (Mars-van Krevelen mechanism).

Hydrogen adsorbed on transition metal surfaces is one of the most important intermediates in a large number of electrocatalytic processes. Hydrogen atoms form moderately strong chemical bonds on transition metal surfaces (Figure 3), thus being very reactive intermediates, but also very sensitive to catalytic poisons [5]. Generally, adsorption energy decreases with an increase in the coverage, due to the repulsive adsorbate-adsorbate interaction and gradual stabilization of metal centres. The experimental and theoretical results for $\mathrm{H}$ adsorption on transition metal surfaces are in satisfactory agreement (Figure 3). The differences between them lie within the energy window of only $0.2 \mathrm{eV}$, which coincides with the accuracy of state of the art computational techniques used in material science studies [37].

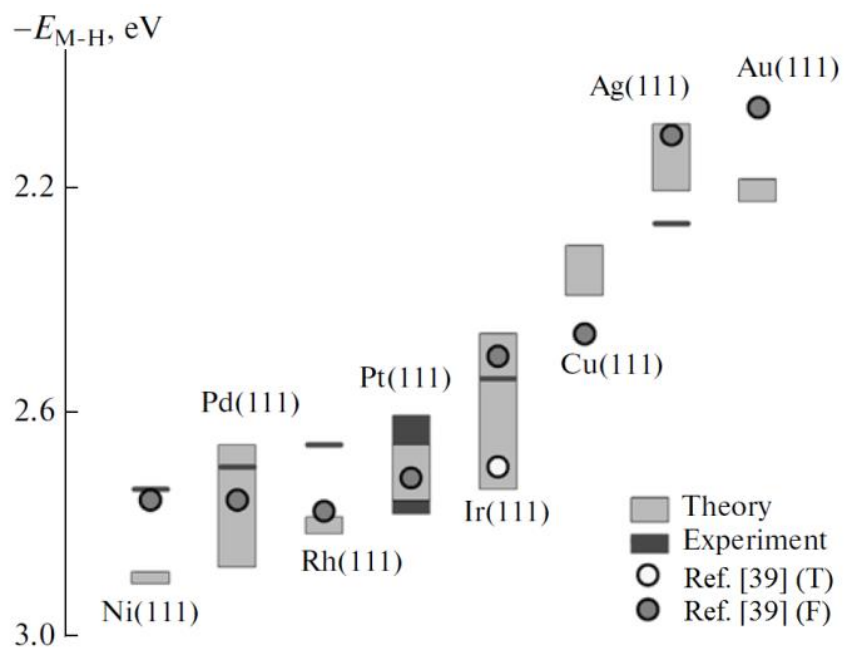

Figure 3. Comparison of $\mathrm{H}$ adsorption energies on transition metal (111) surfaces obtained by both theoretical and experimental methods, in case of $0.25 \mathrm{ML}$ surface coverage ( $T$ - top site, $F$ - three-fold hollow fcc adsorption site). Adapted from ref. [37], ${ }^{\circ}$ 2011, Pleiades Publishing, Ltd.

In the case of HER on metal surfaces in acidic solutions, Trasatti has provided a link between the exchange current density and the metal-hydrogen $(\mathrm{M}-\mathrm{H})$ bond strength, in the form of the volcano curve (Figure 4). Since the highest exchange current density means the highest catalyst activity, the shape of the curve indicates that Pt is the best single-metal catalyst for HER under these conditions [38]. The search for new efficient electrocatalysts is motivated by the high price of Pt. However, it should be noted that around the potential of hydrogen evolution some metals are covered by oxide (W, Mo, etc.), some are covered by underpotential deposited $\mathrm{H}$ (strongly bound hydrogen; metals behaving this way are Pt, Pd, Ir, etc.), while some are reduced, i.e., in metallic state [39]. This concept of HER volcano received significant attention and it is widely accepted. Recently, it was also demonstrated for HER catalyst in alkaline media [40]. However, some new re-interpretations can be found, which also take into account the nature of HER intermediate on different metal surfaces [41]. Even so, formation of $\mathrm{H}_{\text {ads }}$ is the key step in this process.

Another important finding which can be mentioned is related to the scaling of adsorption energies of different molecules and molecular fragments on transition metal surfaces. For the cases of $\mathrm{CH}_{\mathrm{x}}$, $(x \in\{0,1,2,3\}), \mathrm{NH}_{\mathrm{x}}(\mathrm{x} \in\{0,1,2\}), \mathrm{OH}_{\mathrm{x}},(\mathrm{x} \in\{0,1\})$, and $\mathrm{SH}_{\mathrm{x}},(\mathrm{x} \in\{0,1\})$ adsorbed on close-packed and stepped transition-metal surfaces, scaling relations between the adsorption energy of any of the 
molecules considered with the adsorption energy of the corresponding central $(\mathrm{C}, \mathrm{N}, \mathrm{O}$, or $\mathrm{S})$ atom have been found (example shown in Figure 5).
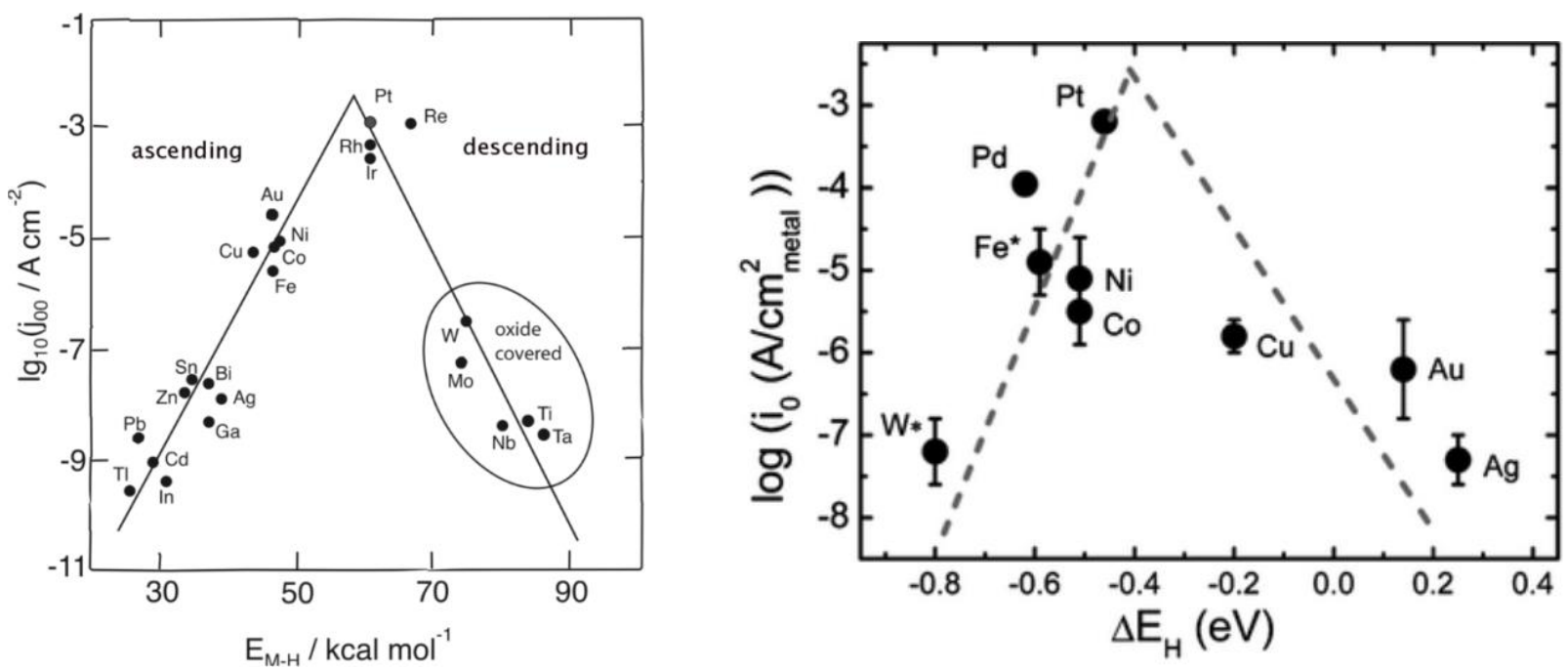

Figure 4. Trasatti's volcano plot for HER in: (left) acidic solutions (joo denotes the exchange current density, and $\mathrm{E}_{M-H}$ the energy of hydride formation) and (right) alkaline solutions $\left(\Delta \mathrm{E}_{H}\right.$ stands for hydrogen binding energy on monometallic surfaces, and i for the exchange current densities). Reprinted from ref. [41], ${ }^{{ }^{0}} 2014$ Quaino et al; licensee Beilstein-Institut; and ref. [42] with permission from The Royal Society of Chemistry

The scaling constant was found to depend only on $x$ [43]. In the light of the adsorption scenarios presented in Figure 4, it is clear that all considered molecular fragments are radical species, so similar adsorption mechanisms are operative for all of them. The power of such relations lies in the fact that with only a few explicitly calculated adsorption energies, one can estimate reaction mechanism of various (electro)catalytic reactions which involve these species.

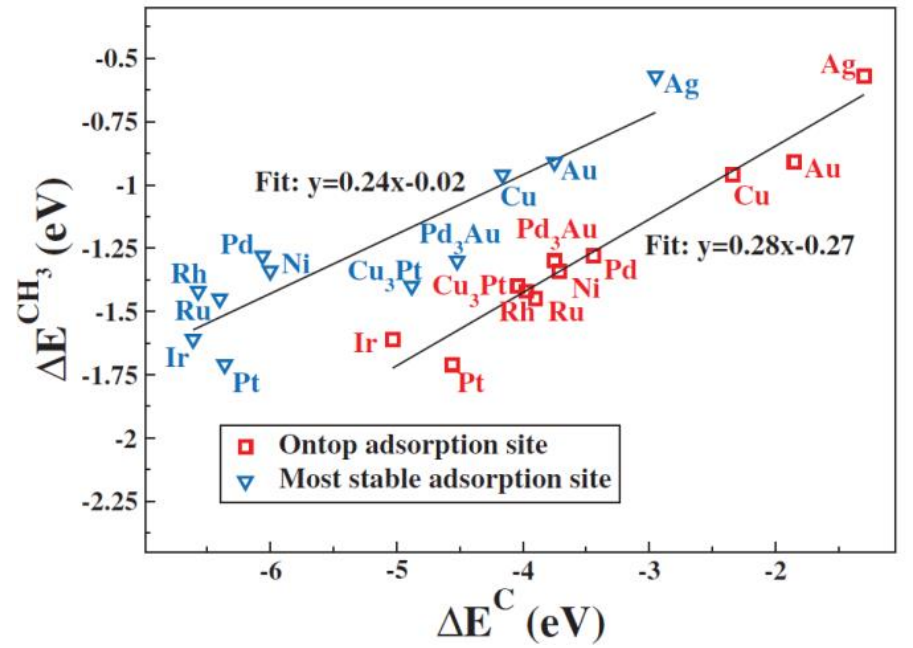

Figure 5. Correlation between the binding energies of $\mathrm{CH}_{3}\left(\Delta \mathrm{E}^{\mathrm{CH}}\right)$ and $\mathrm{C}\left(\Delta \mathrm{E}^{C}\right)$ for adsorption in the most stable sites (triangles) and in the case where both $\mathrm{CH}_{3}$ and $\mathrm{C}$ have been fixed to the on-top site (squares). Reprinted from ref. [43], ${ }^{\circ} 2007$ American Physical Society.

As many of electrocatalytic reactions involve the use of expensive Pt-group metals, one of the strategies to reduce the price is the use of core-shell or thin-layer catalysts [44]. It was recently shown that many properties of such thin films, including chemisorption properties, converge quickly with the thickness of the film and depend only on the lattice mismatch between the overlayer and the support. This means that from the difference between the lattice constants one can estimate chemisorption energies if data are known for pure overlayer [45] (Figure 6). 

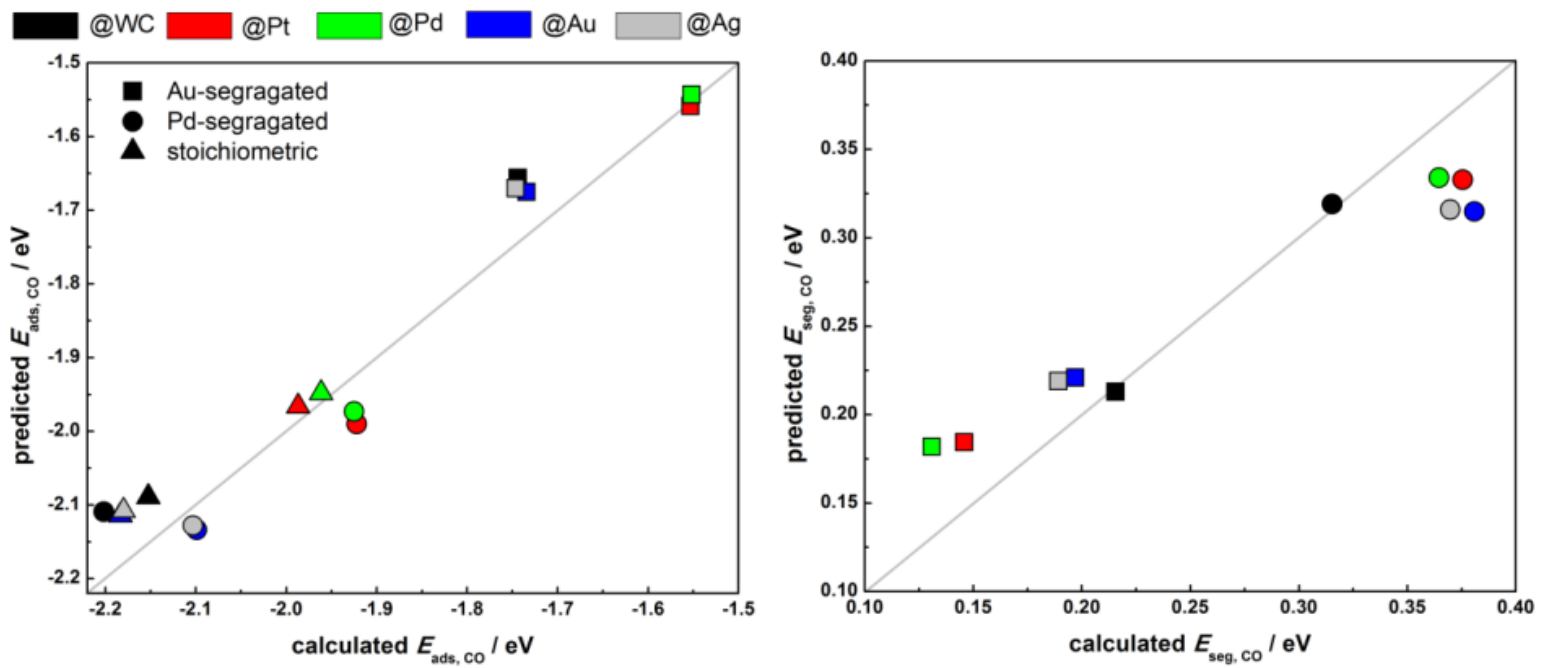

Figure 6. Comparison of the calculated and predicted properties for $\mathrm{Pd}_{3} \mathrm{Au}$ trilayer on different substrates: $\mathrm{CO}$ adsorption energies (left) and surface segregation energies in the presence of CO (right). Surface composition is defined by the symbol, while color defines the substrate (coefficients of determination $\left.R^{2}\left(\mathrm{E}_{a d s, c o}\right)=0.99 ; R^{2}\left(\mathrm{E}_{\text {seg, }, \mathrm{co}}=0.94\right)\right)$. Reproduced from ref. [45] with permission from the PCCP Owner Societies.

If such database is combined with scaling relations, it can be concluded that we already know a lot about the systems that have not been formed yet, even though these systems were not treated explicitly by theoretical calculations. So, the next step is the integration of many existing models and databases in a functional system which can be used to screen electrocatalysts for various reactions.

\section{Chemisorption on metal oxides}

Metal oxides are the most abundant materials in the Earth's crust. They demonstrate a great variability in the crystal and electronic structure, as well as magnetic properties. Surface properties of metal oxides can vary from typical insulator and semiconductor behaviour to metal-like behaviour. Such diversity qualifies them for many technological applications, including those in catalysis [46,47], energy conversion [48], chemical sensors and environmental monitoring [49], corrosion [50], and ceramics [51].

A great variety in oxides properties is the reason why making general remarks about chemisorption trends on metal oxide surfaces is rather difficult, and why the situation is not as clear as in the case of (transition) metal surfaces. As an example, we take magnesium oxide ( $\mathrm{MgO}$ ), although it is an insulator and does not have many electrochemical applications. However, it is one of the most intensively studied oxide materials: its physical properties such as the simple rock salt structure, strong ionic bonding and high chemical stability, make it a perfect candidate for surface and adsorption studies. $\mathrm{MgO}(001)$ surface is especially widely used, as it is non-polar, easy to prepare and does not undergo large structural relaxation [52]. $\mathrm{MgO}$ is quite inert, but its surface can be made more reactive by introducing different types of defect into its structure [53-58], or growing it on a metal substrate [59-62]. Let us consider dissociative adsorption of homonuclear diatomic molecule $\mathrm{X}_{2}$ on $\mathrm{MgO}(001)$, where $\mathrm{X}$ is non-metal from p-block of the Periodic Table of Elements. In order to make a homolytic $\mathrm{X}-\mathrm{X}$ bond break, adsorption should be parallel to the surface plane, so that both $X$ atoms would interact with the surface. The energy spent for $X-X$ bond cleavage is compensated by formation of new surface bonds. Depending on the type of interaction, we can divide such molecules into three groups [53]: (i) weakly interacting with $\mathrm{MgO}\left(\mathrm{H}_{2}, \mathrm{~N}_{2}, \mathrm{O}_{2}\right)$, (ii) strongly adsorbing on $\mathrm{MgO}(001)\left(\mathrm{B}_{2}, \mathrm{C}_{2}\right)$ and (iii) spontaneously dissociating on $\mathrm{MgO}(001)\left(\mathrm{F}_{2}\right)$. Weak 
adsorption is a consequence of very strong intramolecular bonds in these molecules, due to either fully filled bonding states $\left(\mathrm{H}_{2}, \mathrm{~N}_{2}\right)$, or partially filled antibonding states $\left(\mathrm{O}_{2}\right)$ destabilizing the molecule on the surface. $B_{2}$ and $C_{2}$ possess partially filled bonding orbitals located in the same energy window as the $\mathrm{MgO}$ valence band, and these states participate in bonding with $\mathrm{MgO}(001)$ (Figure 7). In case of $F_{2}$ the empty $\sigma^{*}$ orbital located just below $\mathrm{MgO}$ valence band fills upon the interaction with $\mathrm{MgO}$, due to charge transfer to electronegative $\mathrm{F}$ [53]. Hence, it comes to a proper matching between adsorbate and substrate.
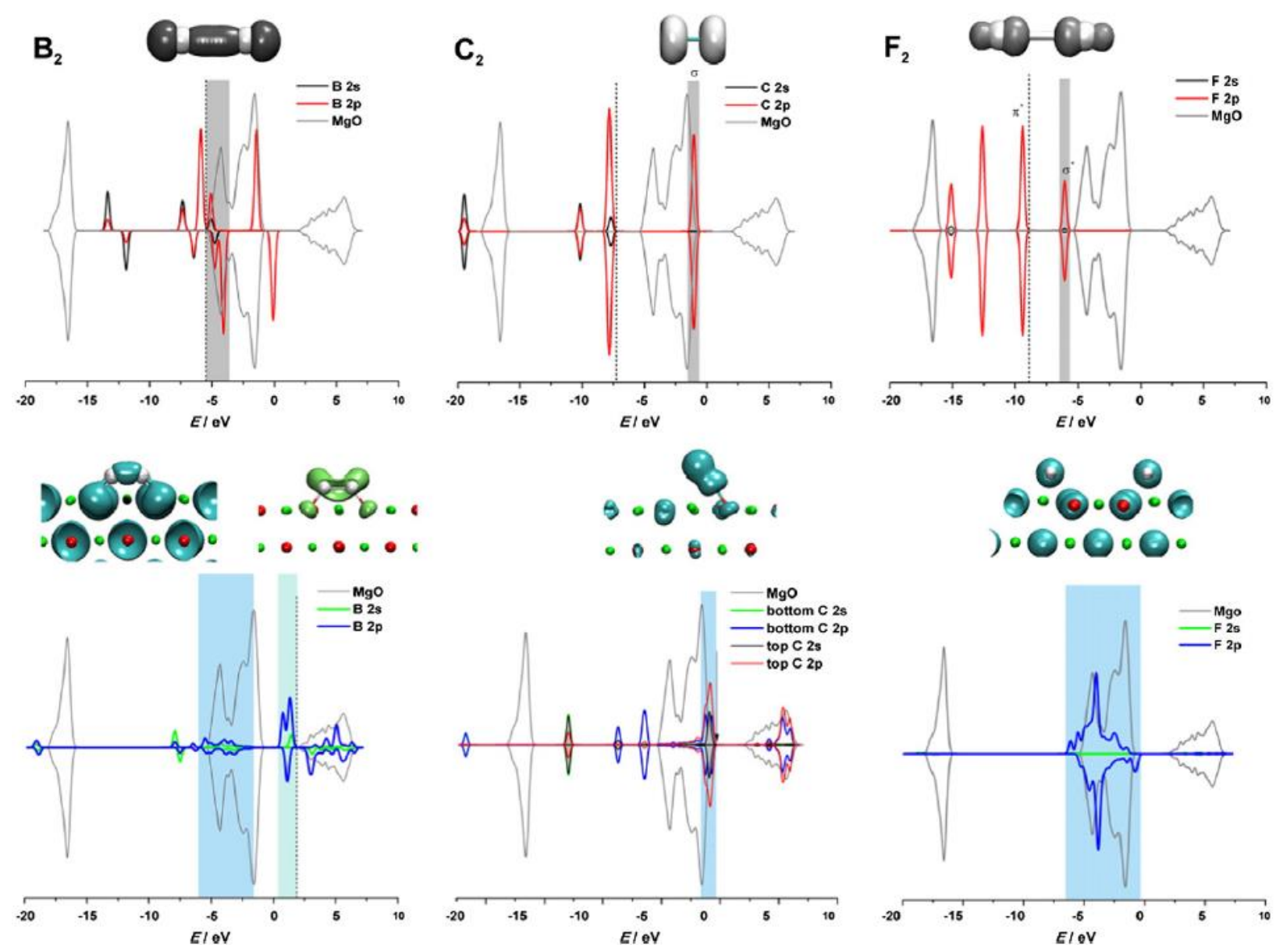

Figure 7. Projected Density of States (PDOS) and Integrated Local Density of States (ILDOS) for isolated (top) and adsorbed (bottom) $B_{2}$ (left), $C_{2}$ (middle) and $F_{2}$ (right). Density of states of clean $\mathrm{MgO}$ (scaled) is included. Vertical dashed line denotes the highest occupied states of investigated molecules (top) or the highest occupied state of $X_{2, a d s}+M g O(001)$ complex. Presented structures give ILDOS in the energy windows designated on PDOS. Reprinted from ref. [53], (C) 2014 Elsevier B.V.

The mechanism of CO oxidation on metal oxide surfaces is generally of Mars-van Krevelen type, as the lattice $\mathrm{O}$ atom is available (Figure 2c). DFT calculations have confirmed this for the case of $\mathrm{Co}_{3} \mathrm{O}_{4}(110)$ surface, where cobalt reduction $\left(\mathrm{Co}^{3+}+\mathrm{e}^{-} \rightarrow \mathrm{Co}^{2+}\right)$ acts as an electron sink, removing electrons upon $\mathrm{CO}_{2}$ formation, and allowing re-oxidation of the surface [63]. Similar was found for a rather good $\mathrm{CO}$ oxidation catalyst, $\mathrm{CeO}_{2}$. As the mechanism assumes vacancy formation in the place of $\mathrm{O}$, the vacancy formation energy can be used as a catalytic descriptor for $\mathrm{CO}$ oxidation reaction [64]. This energy can be tuned by dopants and evaluated by electronic structure theory calculations. That way, one can optimize the catalyst to maximize its activity. Again, Sabatier-like principle is in action: O-vacancy formation energy should be low enough to enable the release of lattice $\mathrm{O}$, but high enough to make the vacancy annihilation by $\mathrm{O}_{2}$ possible [65]. 
Oxide materials have found applications in HER catalysis as well. At the moment, the focus is on interfacial effects where the presence of oxide/hydroxide interface with metal can boost hydrogen evolution through cooperative action in $\mathrm{H}_{2} \mathrm{O}$ activation $[66,67]$. Chemisorption at the interface is the key step here, and the activity trends can be related to the chemisorption of hydrogen and formation of $\mathrm{H}_{\mathrm{ads}}$ at the metallic phase. It also seems that in some cases, oxides could be put on the classical HER volcano curve (Figure 8 ) where $\mathrm{H}_{\text {ads }}$ bond strength with oxide surface can be taken as the descriptor of catalytic activity [68].

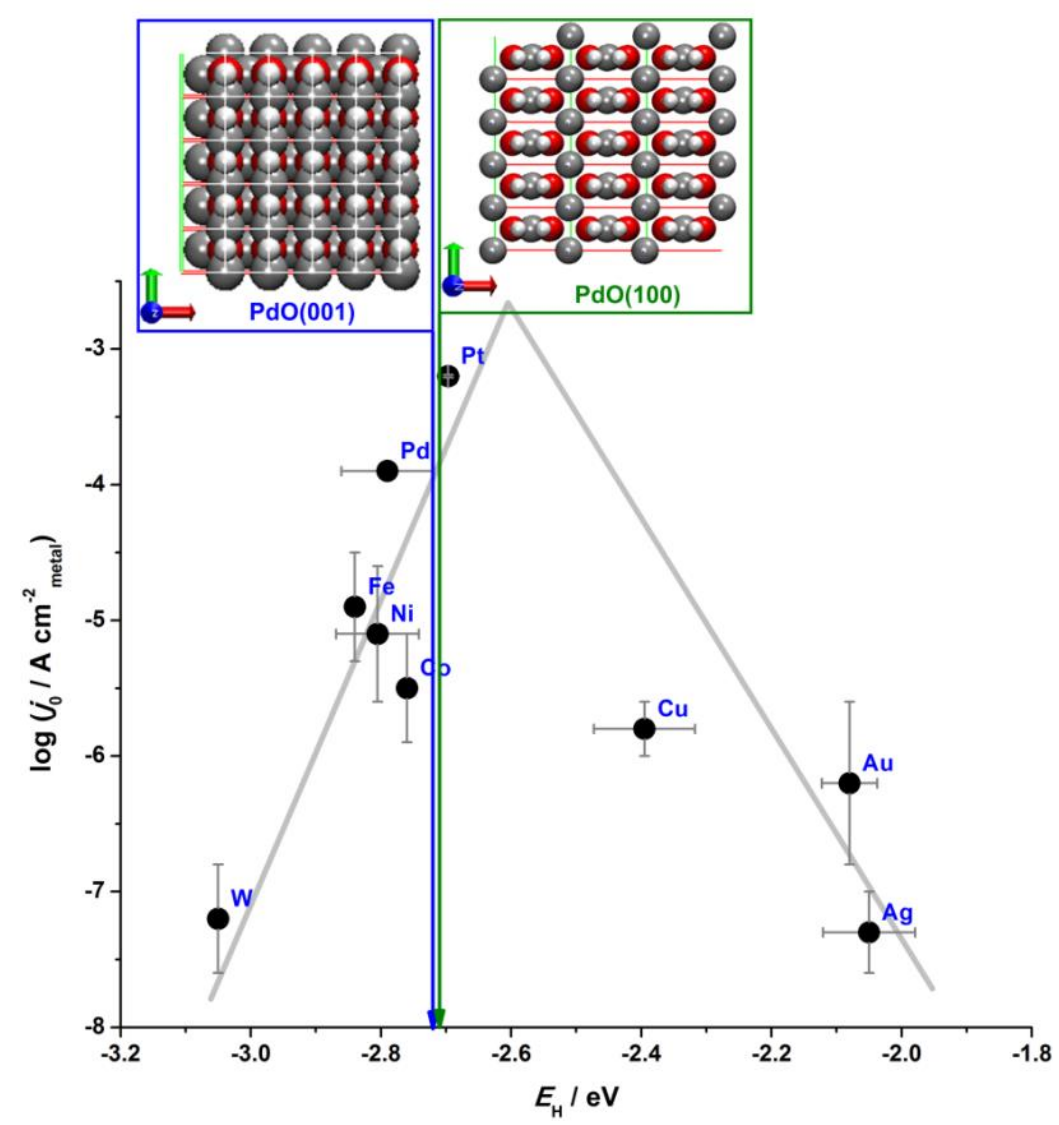

Figure 8. HER volcano curve in alkaline media with PdO located on it (the cases of $1 \mathrm{ML}$ by $\mathrm{H}_{\text {ads }}$ on PdO(001) and PdO(100)). Reprinted from ref. [68], (C) 2017 Elsevier Ltd.

\section{Chemisorption on carbonaceous materials}

Properly functionalized carbon-based materials can be used as either catalysts, or catalyst supports. For example, Li can be inserted reversibly within most carbonaceous materials, but the insertion mechanism depends on the material type - Li intercalates in layered carbons such as graphite, and it adsorbs on the surfaces of single carbon layers. Lithium also appears to reversibly bind near hydrogen atoms in carbonaceous materials containing substantial hydrogen [69]. For catalyst support applications, high conductivity and large specific surface area are of crucial importance. However, modifications might be necessary to achieve good tethering of catalyst nanoparticles to carbon surfaces.

The most basic model for contemplating all carbon-based materials is graphene, a monolayer of carbon atoms arranged in a honeycomb lattice. Since its experimental discovery in 2004, it has become one of the hottest topics in materials science. The 'graphene fever' has particularly influenced the world of electrochemical energy storage devices. Better understanding of graphene reactivity would be useful for such applications, where graphene and other carbon materials are 
key elements [70]. As mentioned before, in order to understand adsorption, it is important to consider electronic structure of both adsorbate and adsorbent.

Carbon atoms in graphene are $\mathrm{sp}^{2}$ hybridized, forming delocalized $\pi$ electronic cloud on both sides of the basal plane. As a result, graphene is chemically inert. Three high symmetry sites are available for atomic adsorption on pristine graphene: top (single-coordinated, above one C atom), bridge (two-coordinated, above the middle of a $\mathrm{C}-\mathrm{C}$ bond) and hollow (six-coordinated, above the centre of $\mathrm{C}_{6}$ hexagon). The nature of graphene interaction with single atoms can be very divergent, depending on the element in question, and detailed data covering most of the Periodic Table of Elements can be found in ref. [70]. For example, sodium preferentially adsorbs on the hollow site, transferring its charge to $C$ atoms consisting $C_{6}$ hollow, in an ionic interaction. As there is no accompanying $\pi$ electronic system disruption nor graphene plane corrugation, such charge transfer results in an upshift of the Fermi level [70]. The situation is similar for other alkali metals, with $\mathrm{ns}^{1}$ charge transfer. For alkaline earth metals ( $\mathrm{ns}^{2}$ electronic configuration) and $\mathrm{Zn}$ (filled $\mathrm{d}$ shell) the interaction is even weaker. On the other hand, atomic hydrogen adsorbs on C-top site, forming a covalent bond with $\mathrm{C}$, causing significant $\mathrm{sp}^{2} \rightarrow \mathrm{sp}^{3}$ re-hybridization of the orbitals of $\mathrm{C}$ atoms surrounding the adsorption site, leading to basal plane deformation [71-73]. Functionalization of the graphene basal plane with O-containing groups has been known to enhance $\mathrm{H}$ adsorption [73], making it comparable in strength to $\mathrm{H}$ adsorption on the coinage metal surfaces [37]. The reason for this enhancement is simple: $\mathrm{O}$-groups introduce local disruptions of $\pi$ electronic cloud of graphene, $\mathrm{sp}^{2} \rightarrow \mathrm{sp}^{3}$ rehybridization of $\mathrm{C}$ atoms to which they are bound, and layer corrugation. Therefore, the first $\mathrm{C}$ neighbours of the $\mathrm{C}-\mathrm{O}$ moieties will be prone to $\mathrm{H}$ bonding. Such strong interaction indicates a possible application of O-functionalized graphene for hydrogen storage. Oxygen functionalities can also improve graphene interaction with alkali metals, but in this case, the group type and concentration are of paramount importance [74,75], as the metal could interact strongly with the group, resulting in phase separation, lethal for electrochemical applications. Scaling between adsorption energies of various simple adsorbates on oxidized graphene surfaces has been demonstrated [73], indicating similar nature of bonding for all of them. Excellent correlations were observed (Figure 9) with the coefficients of determination higher than 0.99 , suggesting that stronger $\mathrm{H}$ adsorption at a given site is, stronger adsorption of other adsorbates at that site will be, as well.

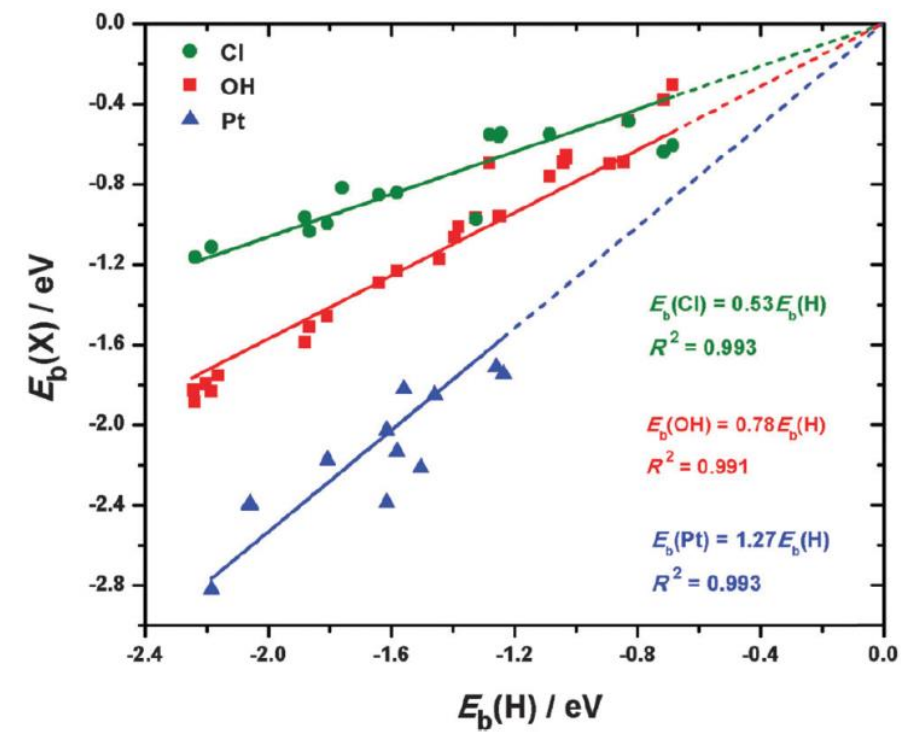

Figure 9. Correlation between $\mathrm{H}$ binding energy and the binding energies of $\mathrm{Cl}, \mathrm{OH}$ and $\mathrm{Pt}$ adsorbates on oxidized graphene surfaces, with corresponding regression lines and coefficients of determination. Reproduced from Ref. [73] with permission from the PCCP Owner Societies 
Scattering of data points is related to the effect of local geometries and the interaction of the adsorbate with $\mathrm{O}$ functional groups instead of the graphene basal plane, as well as different types of adsorption sites preferred by different adsorbates.

When it comes to molecular adsorbates, $\mathrm{O}_{2}$ interacts rather weakly with pristine graphene. However, doping graphene with different heteroatoms like $\mathrm{P}$ or $\mathrm{S}$ significantly strengthens $\mathrm{O}_{2}$ bonding. These two dopants induce layer corrugation and transfer some of their charge to the surrounding $\mathrm{C}$ atoms, becoming in that way slightly positive and attractive for $\mathrm{O}_{2}$. This is, however, not the case with substitutionally introduced nitrogen. In the case of $\mathrm{P}$-doped graphene, $\mathrm{O}-\mathrm{O}$ bond is elongated by approx. $28 \%$ upon adsorption (compared to the bond in the gas phase), while for Sdoped graphene $\mathrm{O}_{2}$ can be considered completely dissociated (Figure 10) [76]. This is precisely the type of molecule activation needed for catalytic purposes. Also, it is a clear example of how alterations of the materials electronic structure affect its applicability for a chosen purpose.

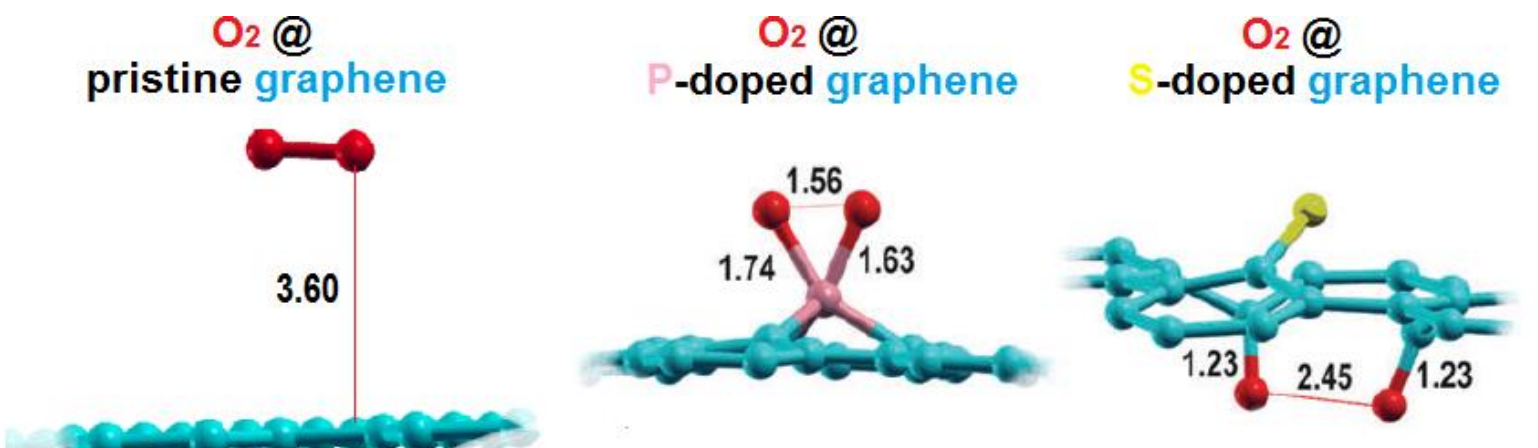

Figure 10. Relaxed structures of $\mathrm{O}_{2}$ adsorbed on P-and S-doped graphene surfaces. Bond lengths are given in angstroms. Adapted from ref. [76] with permission from the PCCP Owner Societies.

\section{Chemisorption on supported metal clusters}

Supported metal nanostructures are the most widely used type of heterogeneous catalyst in industrial processes. For example, Pt nanoparticles supported by carbon are the catalyst of choice for fuel cell applications. Dispersing the active catalyst component over a suitably chosen support enhances its active surface area and the number of active sites, resulting in increased catalytic activity. The size of metal particles is a key factor in determining the performance of such catalysts - the specific activity per metal atom usually increases with decreasing size of the metal particles. Lowering the coordination of a metal atom means leaving it less and less saturated, and therefore more (re)active. However, the surface free energy of metals increases significantly with decreasing particle size, causing aggregation of clusters. Sometimes, the support can also contribute to the catalytic activity (the so-called active support). In such cases the interface between the support and the catalyst particle is also of great importance.

Although the macroscopic noble metals are inert and not susceptible to oxidation, their nano-sized forms are often very reactive and excellent catalysts. For example, Pt-cluster size strongly influences $\mathrm{CO}$ adsorption energy (Figure 11). It is similar with Au: when moving from the densely packed (111) surface to 12-atoms cluster, Au evolves from inactive to the most active material towards $\mathrm{CO}$ oxidation. A detailed DFT study of $\mathrm{CO}$ oxidation on Au surfaces suggested that the oxidation of $\mathrm{CO}$ on Au nanoparticles dispersed over an inactive support occurs on Au-steps in two consecutive stages: (i) $\mathrm{CO}+\mathrm{O}_{2} \rightarrow \mathrm{CO}_{2}+\mathrm{O}$, and (ii) $\mathrm{CO}+\mathrm{O} \rightarrow \mathrm{CO}_{2}$ [77]. In the case of active support, $\mathrm{CO}$ oxidation follows the same steps occurring at Au-support interface. From the mechanism, it is clear that the supported $\mathrm{Au}$ cluster must be large enough to enable the co-adsorption of $\mathrm{CO}$ and $\mathrm{O}_{2}$. In the case of MgO-support, DFT calculations suggest that this is possible starting already with Au-trimers [46]. 


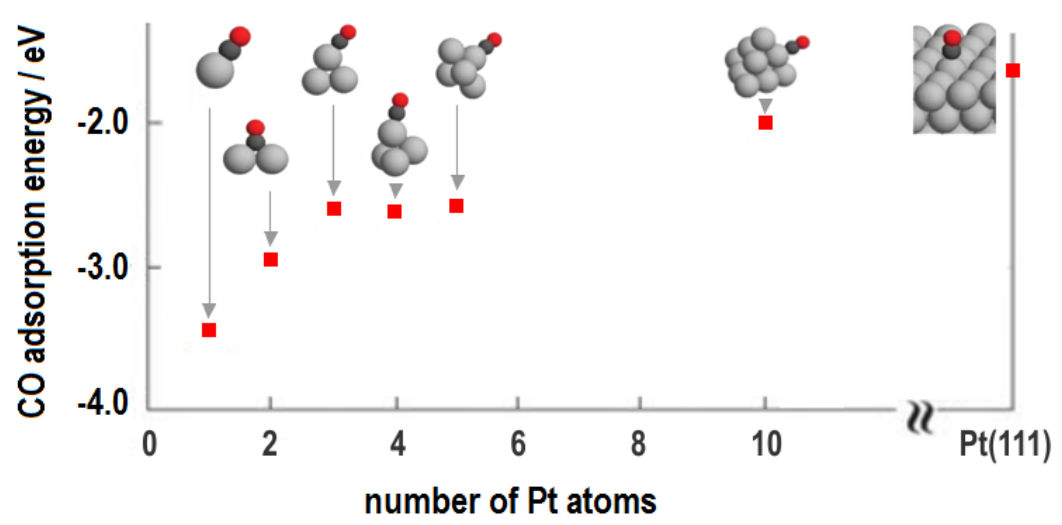

Figure 11. Influence of Pt-cluster size on-DFT-calculated CO adsorption energy, with corresponding adsorption structures given as insets. Adapted from ref. [78], with permission from PCCP Owner Societies

It is clear that very small metal clusters are in fact molecular systems and until their size is large enough (typically a few $\mathrm{nm}$ ) it is not possible to talk about band structure, so the models used for transition metals do not necessarily work in these cases. Also, there is a large number of low coordinated atoms which are often much more reactive than regular terrace sites. In these cases, XES and XAS have been shown as extremely useful techniques which provide information about local bonding and valuable input for theoretical calculations. Unfortunately, computational resources which are typically available do not allow exact treatments of nanoparticles (as they count thousands of atoms) and in order to model such systems one must simplify the model to obtain data regarding the adsorption of reactants and intermediates of investigated (electro)catalytic reaction.

\section{Chemisorption on supported single atoms}

Talking about supported metal clusters, we have mentioned that the surface free energy of metals increases significantly with decreasing particle size, promoting aggregation of metal atoms into small clusters. However, if the support material interacts with metal atoms strongly enough it will prevent the aggregation. The down limit is the single-atom catalyst (SAC), with isolated metal atoms dispersed on an appropriate support (Figure 12). SACs minimize the amount of the metal necessary for good catalytic activity, which is particularly important for supported noble metal catalysts, due to the abundance and price of metals. SACs offer great potential for achieving high catalytic activity, where isolated metal atoms anchored to support can act as active centres.

a

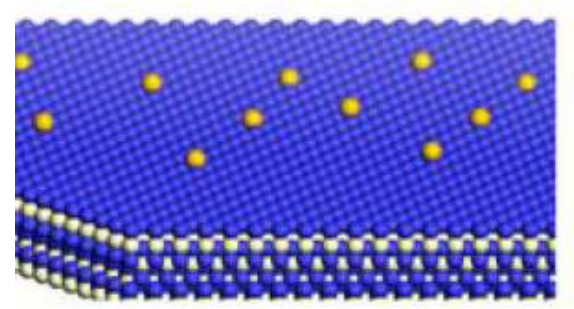

b

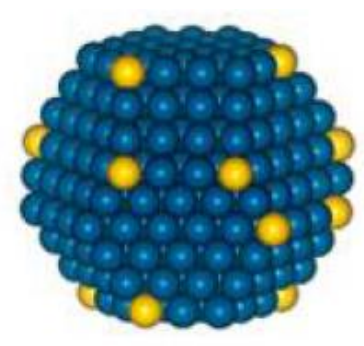

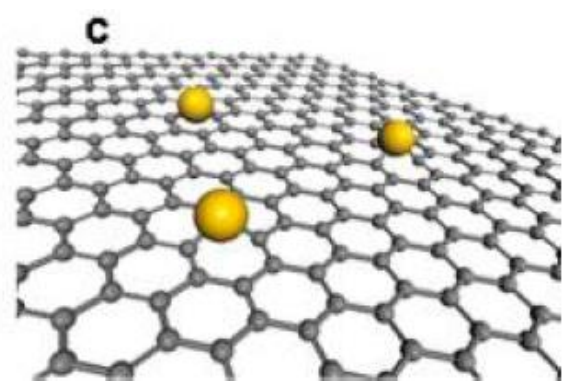

Figure 12. Example structures of different types of SACs: single metal atoms (yellow) supported by (a) metal oxide, (b) metal surfaces, and (c) graphene. Reprinted (adapted) with permission from ref. [79]. Copyright (2013) American Chemical Society

Single supported metal atoms possess unique electronic properties different from those of metal nanocatalysts. The coordination of the single atom to the surface atoms of the support is critical, 
and it cannot be discussed as an isolated single metal atom without the support. The bonding of metal atom to the support leads to charge transfer between metal atoms and the support due to different chemical potentials [79]. As a result, the anchored metal atom usually carries some charge, as was shown by various spectral measurements and computational studies. Obviously, chemisorption is of crucial importance for SACs. First, strong-enough anchoring (to prevent clustering) of the metal to the support is needed, and next, optimal strength chemisorption of reactants/intermediates of the chosen catalytic reaction is necessary to provide good catalytic activity of SAC. For now, there is no universal catalytic activity descriptor for SACs.

\section{Conclusions}

Chemisorption at electrified interface is the crucial step in virtually every electrochemical energy conversion process. Hence, in order to progress further in materials development, we must understand how reactants and intermediates interact with electrodes. So far, a lot has been done for the case of metal surface, but much less is known regarding general trends in adsorption on metal oxides and carbon materials. To make the situation worse, realistic systems like supported metal clusters are extremely complicated to be treated as whole using modern computational techniques, while single atom catalysts cannot be considered separately from the support. For this reason, theory and experiment must be closely synchronized to provide atomic level understanding of chemisorption in energy conversion processes.

Acknowledgements: This work has been performed under the NATO SPS multi-year project G5729 "Optimizing Fuel Cell Catalyst Stability upon Integration with Reforming - OFICeR". Authors acknowledge the support provided by the Serbian Ministry of Education, Science and Technological Development (grant no. III45014).

\section{References}

[1] J. G. Calvert, Pure and Applied Chemistry 62 (1990) 2167-2219.

[2] D. H. Everett, Pure and Applied Chemistry 31 (1972) 577-638.

[3] R. L. Burwell, Advances in Catalysis 26 (1977) 351-392.

[4] B. E. Conway, Electrochemical Supercapacitors: Scientific Fundamentals and Technological Applications, Springer Science \& Business Media, 1999.

[5] N. M. Marković, P. N. Ross, Surface Science Reports 45 (2002) 117-229.

[6] P. Sabatier, Berichte der Deutchen Chemischen Gesselschaft 44 (1911) 1984-2001.

[7] M. Armand, J.-M. Tarascon, Nature 451 (2008) 652-657.

[8] J.-Y. Hwang, S.-T. Myung, Y.-K. Sun, Chemical Society Reviews 46 (2017) 3529-3614.

[9] D. Aurbach, H. Gizbar, A. Schechter, O. Chusid, H.E. Gottlieb, Y. Gofer, I. Goldberg, Journal of the Electrochemical Society 149 (2002) A115-A121.

[10] J. V. Rani, V. Kanakaiah, T. Dadmal, M. S. Rao, S. Bhavanarushi, Journal of the Electrochemical Society 160 (2013) A1781-A1784.

[11] I.A. Pašti, N. V. Skorodumova, S. V. Mentus, Reaction Kinetics, Mechanisms and Catalysis 115 (2015) 5-32.

[12] A. Bajpai, M. Rajpoot, Journal of Scientific and Industrial Research 58 (1999) 844-860.

[13] A. Nilsson, L.G.M. Pettersson, Surface Science Reports 55 (2004) 49-167.

[14] A. Nilsson, M. Weinelt, T. Wiell, P. Bennich, O. Karis, N. Wassdahl, J. Stöhr, M. G. Samant, Physical Review Letters 78 (1997) 2847-2850.

[15] A. Nilsson, J. Hasselström, A. Föhlisch, O. Karis, L. G. M. Pettersson, M. Nyberg, L. Triguero, Journal of Electron Spectroscopy and Related Phenomena 110-111 (2000) 15-39.

[16] H. Gross, C. T. Campbell, D. A. King, Surface Science 572 (2004) 179-190.

[17] P. Hohenberg, W. Kohn, Physical Review 136 (1964) B864-B871. 
[18] W. Kohn, Fundamentals of density functional theory, in: Density Funct. Theory Appl., Springer Berlin Heidelberg, 1998: pp. 1-7.

[19] M. D. Segall, P. J. D. Lindan, M. J. Probert, C. J. Pickard, P. J. Hasnip, S. J. Clark, M. C. Payne, Journal of Physics: Condensed Matter 14 (2002) 2717-2744.

[20] D. W. Boukhvalov, Y. Wu, Y. Hao, J. Geng, M. Charlton, S. Chen, Y. Ren, H. Ji, H. Li, D. W. Boukhvalov, R. D. Piner, C. W. Bielawski, R. S. Ruoff, K. S. Novoselov, L. A. Ponomarenko, RSC Advances 3 (2013) 7150.

[21] J. Dai, J. Yuan, Physical Review B 81 (2010) 165414.

[22] E. Santos, P. Quaino, W. Schmickler, Physical Chemistry Chemical Physics 14 (2012) 11224-11233.

[23] J. Greeley, J. Rossmeisl, Zeitschrift Für Physikalische Chemie 221 (2007) 1209-1220.

[24] M. E. Björketun, A. S. Bondarenko, B. L. Abrams, I. Chorkendorff, J. Rossmeisl, Physical Chemistry Chemical Physics 12 (2010) 10536.

[25] J. K. Nørskov, F. Abild-Pedersen, F. Studt, T. Bligaard, Proceedings of the National Academy of Sciences of the United States of America 108 (2011) 937-43.

[26] B. Hammer, J.K. Norskov, Nature 376 (1995) 238-240.

[27] V. R. Stamenkovic, B. Fowler, B. S. Mun, G. Wang, P. N. Ross, C. A. Lucas, N. M. Markovic, Science 315 (2007) 493-497.

[28] F. H. B. Lima, J. Zhang, M. H. Shao, K. Sasaki, M. B. Vukmirovic, E. A. Ticianelli, R. R. Adzic, Journal of Physical Chemistry C 111 (2007) 404-410.

[29] A. Ruban, B. Hammer, P. Stoltze, H. L. Skriver, J. K. Nørskov, Journal of Molecular Catalysis A: Chemical 115 (1997) 421-429.

[30] L. G. M. Pettersson, A. Nilsson, Topics in Catalysis 57 (2014) 2-13.

[31] A. Nilsson, L. G. M. Pettersson, J. K. Nørskov, Chemical Bonding at Surfaces and Interfaces, Elsevier, Amsterdam, 2008.

[32] J. S. Dewar, Bulletin de La Societe Chimique de France 18 (1951) C71-C79.

[33] J. Chatt, L. A. Duncanson, Journal of the Chemical Society (Resumed) (1953) 2939-2947.

[34] T. Engel, G. Ertl, Advances in Catalysis 28 (1979) 1-78.

[35] B. L. M. Hendriksen, J. W. M. Frenken, Physical Review Letters 89 (2002) 046101.

[36] B. L. M. Hendriksen, S. C. Bobaru, J. W. M. Frenken, Surface Science 552 (2004) $229-242$.

[37] D. Vasić, Z. Ristanović, I. Pašti, S. Mentus, Russian Journal of Physical Chemistry A 85 (2011) 23732379.

[38] S. Trasatti, Journal of Electroanalytical Chemistry and Interfacial Electrochemistry 39 (1972) 163184.

[39] B. E. Conway, Progress in Surface Science 49 (1995) 331-452.

[40] D. Zhao, Q. Shao, Y. Zhang, X. Huang, Nanoscale 10 (2018) 22787-22791.

[41] P. Quaino, F. Juarez, E. Santos, W. Schmickler, Beilstein Journal of Nanotechnology 5 (2014) 846854.

[42] W. Sheng, M. Myint, J. G. Chen, Y. Yan, D. M. Kolb, R. J. Behm, I. Chorkendorff, J. K. Norskov, Energy \& Environmental Science 6 (2013) 1509.

[43] F. Abild-Pedersen, J. Greeley, F. Studt, J. Rossmeisl, T. R. Munter, P. G. Moses, E. Skúlason, T. Bligaard, J.K. Nørskov, Physical Review Letters 99 (2007) 016105.

[44] I. A. Pašti, E. Fako, A. S. Dobrota, N. López, N. V. Skorodumova, S. V. Mentus, ACS Catalysis 9 (2019) 3467-3481.

[45] E. Fako, A. S. Dobrota, I. A. Pašti, N. López, S. V. Mentus, N. V. Skorodumova, Physical Chemistry Chemical Physics 20 (2018) 1524-1530.

[46] M. Amft, N. V. Skorodumova, Physical Review B - Condensed Matter and Materials Physics 81 (2010) 195443.

[47] B. Yoon, H. Häkkinen, U. Landman, A. S. Wörz, J. M. Antonietti, S. Abbet, K. Judai, U. Heiz, Science 307 (2005) 403-407.

[48] P. Poizot, S. Laruelle, S. Grugeon, L. Dupont, J.-M. Tarascon, Nature 407 (2000) 496-499.

[49] G. F. Fine, L. M. Cavanagh, A. Afonja, R. Binions, Sensors 10 (2010) 5469-5502.

[50] T. Lei, C. Ouyang, W. Tang, L.-F. Li, L.-S. Zhou, Surface and Coatings Technology 204 (2010) 37983803.

[51] M. Matsuoka, Japanese Journal of Applied Physics 10 (1971) 736-746. 
[52] M. Ménétrey, A. Markovits, C. Minot, Surface Science 566-568 (2004) 693-697.

[53] I. A. Pašti, M. Baljozović, N. V Skorodumova, Surface Science 632 (2015) 39-49.

[54] I. A. Pašti, N. V. Skorodumova, Physical Chemistry Chemical Physics 18 (2016) 426-435.

[55] S. Fernandez, A. Markovits, C. Minot, The Journal of Physical Chemistry C 112 (2008) 16491-16496.

[56] C.A. Scamehorn, N. M. Harrison, M. I. McCarthy, The Journal of Chemical Physics 101 (1994) 15471554.

[57] R. Nada, A.C. Hess, C. Pisani, Surface Science 336 (1995) 353-361.

[58] M. J. Stirniman, C. Huang, R. Scott Smith, S. A. Joyce, B. D. Kay, The Journal of Chemical Physics 105 (1996) 1295-1298.

[59] G. Pacchioni, N. Rösch, The Journal of Chemical Physics 104 (1996) 7329-7337.

[60] P. A. Žguns, M. Wessel, N. V. Skorodumova, RSC Advances 5 (2015) 94436-94445.

[61] H. J. Zhai, J. Döbler, J. Sauer, L. S. Wang, Journal of the American Chemical Society 129 (2007) 13270-13276.

[62] H. J. Freund, G. Pacchioni, Chemical Society Reviews 37 (2008) 2224-2242.

[63] P. Broqvist, I. Panas, H. Persson, Journal of Catalysis 210 (2002) 198-206.

[64] M. Aryanpour, A. Khetan, H. Pitsch, ACS Catalysis 3 (2013) 1253-1262.

[65] V. Shapovalov, H. Metiu, Journal of Catalysis 245 (2007) 205-214.

[66] R. Subbaraman, D. Tripkovic, D. Strmcnik, K. C. Chang, M. Uchimura, A. P. Paulikas, V. Stamenkovic, N. M. Markovic, Science 334 (2011) 1256-1260.

[67] N. Danilovic, R. Subbaraman, D. Strmcnik, K.-C. Chang, A. P. Paulikas, V. R. Stamenkovic, N. M. Markovic, Angewandte Chemie International Edition 51 (2012) 12495-12498.

[68] M. Vasić, M. Čebela, I. Pašti, L. Amaral, R. Hercigonja, D. M. F. Santos, B. Šljukić, Electrochimica Acta 259 (2018) 882-892.

[69] K. Sato, M. Noguchi, A. Demachi, N. Oki, M. Endo, Science 264 (1994) 556-558.

[70] I. A. Pašti, A. Jovanović, A. S. Dobrota, S. V. Mentus, B. Johansson, N. V. Skorodumova, Applied Surface Science 436 (2018) 433-440.

[71] S. Casolo, O. M. Løvvik, R. Martinazzo, G. F. Tantardini, The Journal of Chemical Physics 130 (2009) 054704.

[72] P. A. Denis, F. Iribarne, Journal of Molecular Structure: THEOCHEM 907 (2009) 93-103.

[73] A. S. Dobrota, I. A. Pašti, S. V. Mentus, N. V. Skorodumova, Physical Chemistry Chemical Physics 18 (2016) 6580-6586.

[74] A. S. Dobrota, I. A. Pašti, N. V. Skorodumova, Electrochimica Acta 176 (2015) 1092-1099.

[75] A. S. Dobrota, S. Gutić, A. Kalijadis, M. Baljozović, S. V. Mentus, N. V. Skorodumova, I. A. Pašti, RSC Advances 6 (2016) 57910-57919.

[76] A. S. Dobrota, I. A. Pašti, S. V. Mentus, N. V. Skorodumova, Physical Chemistry Chemical Physics 19 (2017) 8530-8540.

[77] Y.-L. Song, L.-L. Yin, J. Zhang, P. Hu, X.-Q. Gong, G. Lu, Surface Science 618 (2013) 140-147.

[78] Y. Xu, R.B. Getman, W. A. Shelton, W. F. Schneider, Physical Chemistry Chemical Physics 10 (2008) 6009.

[79] X.-F. Yang, A. Wang, B. Qiao, J. Li, J. Liu, T. Zhang, Accounts of Chemical Research 46 (2013) 17401748.

(C2020 by the authors; licensee IAPC, Zagreb, Croatia. This article is an open-access article distributed under the terms and conditions of the Creative Commons Attribution license (http://creativecommons. org/licenses/by/4.0/) 